\title{
CRIMEN SIN CASTIGO. IMPUNIDAD EN AMÉRICA LATINA
}

\author{
Esteban Beltrán*
}

Todos los días, Amnistía Internacional tiene conocimiento de nuevas violaciones graves de los derechos humanos, como homicidios políticos y «desapariciones», cometidas en diferentes partes del mundo. Uno de los principales factores que contribuye a esta realidad inhumana es el fenómeno de la impunidad. Mientras los agentes de la represión crean que pueden secuestrar, torturar y asesinar sin temor a ser descubiertos o castigados, el ciclo de la violencia no terminará jamás.

A veces, en derechos humanos, la frialdad estadística nos permite imaginar la magnitud de las violaciones a los mismos. En el año 1997, en más de 55 países se llevaron a cabo ejecuciones extrajudiciales y en 31 países hubo "desapariciones" o personas "desaparecidas", en años anteriores siguieron en paradero desconocido. Las fuerzas de seguridad de 117 países sometieron a malos tratos o torturas a personas en sus manos. Todavía hoy en 87 países hay presos de conciencia o posibles presos de conciencia. Todavía hoy, en 53 países, se arrestó y detuvo arbitrariamente a personas o se las tuvo privadas de libertad sin juzgarlas. También, durante 1997 , se llevaron a cabo ejecuciones en 40 países.

Pese a lo demoledor de estos datos, con los espectaculares cambios políticos ocurridos en varias partes del mundo, los derechos humanos han alcanzado mayor relieve que nunca en las relaciones internacionales. Para los gobiernos cada vez es más embarazoso ser tildado de violador de los derechos humanos, no sólo en las Naciones Unidas sino en otras relaciones entre los Estados. En junio de 1993 se celebró la segunda Conferencia Mundial de Derechos Humanos en Viena, Austria. Estuvieron representados más de 170 gobiernos, y con diferente nivel de sinceridad, reafirmaron solemnemente su compromiso de cumplir su obligación de respetar y promover los derechos humanos.

Países cuyos gobernantes hace una década ni siquiera fingían respetar los derechos humanos, en la actualidad proclaman su importancia. Algunos están adaptando sus leyes a las normas internacionales de derechos humanos o al menos han prometido hacerlo. Muchos han creado nuevas instituciones para promover y proteger los derechos humanos. En algunos casos, esto refleja un compromiso real de los nuevos gobiernos. En otros, es simplemente un gesto carente de voluntad política clara.
Sin embargo, los homicidios políticos, la tortura, las «desapariciones», los arrestos arbitrarios y las detenciones continúan cometiéndose impunemente, en ocasiones en una escala espantosa. En algunos de los países en los que se cometen violaciones más brutales de derechos humanos sigue sin intentarse seriamente que los responsables rindan cuentas.

Actualmente están aumentando las violaciones graves de derechos humanos cometidas en países con gobiernos elegidos en las urnas que se han comprometido expresamente con los derechos humanos y las instituciones. El abismo entre los compromisos y la práctica sólo puede salvarse si los abusos contra los derechos humanos se investigan adecuadamente y los responsables quedan a disposición judicial. Las víctimas, sus familiares y la sociedad en general tienen un interés vital en conocer la verdad sobre los abusos. Poner a disposición judicial a los responsables no sólo es importante en los casos individuales, sino que transmite un mensaje claro de que las violaciones de derechos humanos no se tolerarán y que los que las perpetren deberán rendir cuentas. Cuando se permite que los agentes de la policía y las fuerzas de seguridad cometan delitos impunemente, florecen actitudes nocivas y el desprecio por el respeto de la ley.

Para proteger los derechos básicos de las personas, debemos contar con un sistema internacional de justicia que complemente a los tribunales nacionales existentes. Amnistía Internacional, en este sentido, considera históricas dos decisiones que pueden ayudar a romper este círculo de impunidad. En primer lugar la decisión la Audiencia Nacional de España de que es competente para investigar delitos como el genocidio independientemente de la nacionalidad de la víctima y del lugar donde haya podido perpetrarse y, también, la creación de un Tribunal Penal Internacional, que permitirá, cuando se constituya, llevar a juicio a los presuntos responsables de crímenes contra la humanidad, entre otros delitos.

\section{Latinoamérica}

En algunos países, las violaciones las cometen grupos paramilitares o los denominados «escuadrones de la muerte» que actúan con permiso oficial. 
Los gobiernos suelen afirman que son impotentes para controlar a los grupos paramilitares y se niegan a aceptar su responsabilidad de poner fin a estos abusos. En Colombia, muchas violaciones de los derechos humanos cometidas en los últimos años, entre ellas miles de homicidios políticos y centenares de «desapariciones», se han atribuido a las organizaciones paramilitares. Las autoridades colombianas las han descrito como grupos de extremistas de derechas que actúan al margen del control del Estado. Sin embargo, las investigaciones judiciales han descubierto indicios irrefutables de que muchos grupos paramilitares están integrados o cuentan con el respaldo de miembros de las fuerzas armadas colombianas.

En Brasil, cada año, centenares de personas mueren a manos de la policía y de escuadrones de la muerte vinculados a las fuerzas de seguridad, en circunstancias que indicaban que habían sido ejecutadas extrajudicialmente. Las autoridades del estado de Río de Janeiro ofrecen, desde el año 1995, a los policías, aumentos de sueldo por participar en acciones de "valentía y arrojo". Un informe elaborado por las autoridades legislativas estableció que entre enero y julio de 1996 la policía había matado a 942 personas, la mayor parte de ellas muertas por disparos en la cabeza o en la espalda.

En México, durante los últimos años decenas de personas han "desaparecido" o han sido ejecutadas extrajudicialmente a manos de miembros de las fuerzas de seguridad.

Tampoco han desaparecido de Latinoamérica la práctica de la tortura y los malos tratos o el encarcelamiento de personas que se consideran presos de conciencia. En México, por ejemplo, la tortura y los malos tratos se emplean de forma generalizada. En Perú, por ejemplo, se sigue practicando la tortura incluso contra miembros de las fuerzas de seguridad que denuncian esta práctica. A principios del año 1997, Leonor La Rosa Bustamante, agente del servicio de inteligencia del ejército y sospechosa de filtrar a la prensa planes de seguridad, fue torturada en el sótano de unas instalaciones militares de Lima donde, según dijo, también se torturaba a miembros de los grupos alzados en armas.

En Chile, a principios de febrero de este año, varias decenas de presos políticos fueron golpeados $\mathrm{y}$, al menos tres de ellos, sufrieron torturas con la picana eléctrica que descarga electricidad de alto voltaje sobre los cuerpos de las personas torturadas.

En Cuba, México y Perú, por ejemplo, centenares de personas permanecen detenidas por sus opiniones políticas o sus actividades pacíficas de oposición al gobierno o, como en el caso de Perú, son personas inocentes acusadas de colaborar con grupos armados, detenidas por meses o años y condenadas en juicios que no cumplen las garantías internacionales para un juicio justo.

\section{Investigaciones}

Una técnica empleada por los gobiernos y los departamentos de las fuerzas de seguridad para desviar las críticas es anunciar una investigación que posteriormente no lleva a ninguna parte. Algunas veces, los anuncios se hacen de buena $\mathrm{fe}$, pero si no consiguen resultados terminan actuando simplemente como una barrera contra la verdad. En los casos reiterados de matanzas perpetradas por la policía en algunos estados brasileños, Amnistía Internacional ha instado a las autoridades federales a que se encarguen de las investigaciones para impedir la obstrucción, la manipulación y la pérdida de testimonios vitales en el curso de las investigaciones realizadas por la policía de los estados.

Incluso cuando se llevan a cabo las investigaciones y se inician los procedimientos judiciales, las instituciones responsables de la administración de justicia son muchas veces débiles o ineficaces. Frecuentemente, son objeto de presiones de otros departamentos del gobierno o de las fuerzas de seguridad. Los fiscales o los jueces en ocasiones se comportan con un valor admirable, sólo para que les maten o tengan que huir debido a que sus gobiernos no están dispuestos o no son capaces de protegerlos de las amenazas de los acusados, normalmente agentes de sus propias fuerzas de seguridad. En Guatemala, la juez María Eugenia Villaseñor, juez de la cámara de apelaciones, escribió un libro crítico con respecto a la forma en que el sistema judicial se había ocupado del caso de Myrna Mack, antropóloga estadounidense muerta en 1990. Como resultado de esto y de su participación en otras causas relacionadas con violaciones de derechos humanos, la juez Villaseñor ha sufrido reiteradas amenazas de muerte, actos de intimidación y, durante una temporada, tuvo que huir del país.

Hace apenas unos días, la Comisión para el Esclarecimiento Histórico de Guatemala publicó su informe "Memoria del silencio" en el cual ha documentado mas de 600 masacres durante el conflicto de más de 30 años, y que decenas de miles de personas, especialmente indígenas, fueron masacradas en una política de genocidio que fue del año 1981 al año 1983. Si se cumplen las recomendaciones de la Comisión habrá una posibilidad de que la verdad y la justicia cimenten una nueva sociedad en Guatemala.

\section{El proceso judicial}

En ocasiones, el proceso judicial se ve subvertido por medidas como transferir las causas a las cortes especiales. Habitualmente, como en Colombia y Perú, se trata de cortes militares que pocas veces intentan procesar firmemente a compañeros oficiales en causas por violaciones de derechos humanos. Los encausados suelen ser absueltos, en ocasiones, hasta ascendidos. Mientras era investigado por su participación en una matanza de 21 trabajadores de una plantación de plátanos en la región de Urabá, Colombia, ocurrida en 1988, el comandante del ejército colombiano Luis Felipe Becerra fue ascendido a teniente coronel. Le absolvieron a pesar de los fuertes indicios sobre su participación en la matanza, y volvió a verse implicado directamente en la matanza de otros 13 campesinos en 1993. Si hubiera rendido cuentas de sus delitos anteriores, esas 13 personas podrían estar hoy vivas.

En los casos excepcionales en que se consiguen condenas, las penas rara vez guardan proporción con el crimen.

Las leyes de amnistía que impiden los procesamientos, los juicios y las investigaciones minuciosas socavan la búsqueda de la verdad y la justicia. Algunos regímenes responsables de violaciones de derechos humanos que preveían que 
iban a perder el poder se han anticipado a las investigaciones y han proclamado amnistías. Los gobiernos posteriores han sufrido enormes presiones para que ratificaran tales medidas o para que las concedieran si no se había hecho ya.

\section{Defensores de los derechos humanos}

Para Amnistía Internacional constituye motivo de gran preocupación las amenazas reiteradas, el hostigamiento, la detención arbitraria y la tortura que los agentes del Estado infligen a los defensores de derechos humanos. Muchos han sido objeto de «desaparición», muchos han muerto.

Los defensores desempeñan una labor fundamental en los países que padecen las consecuencias de un conflicto violento o una dictadura, en los que los agentes del Estado se sienten libres para actuar impunemente. Muchas veces, son la única fuerza que se interpone entre la mayoría de los ciudadanos normales y el poder desbocado del Estado. Proceden de todos los campos: pueden ser profesores de universidad, periodistas, sacerdotes, dirigentes agrarios o familiares de víctimas. A pesar de la persecución de la que suelen ser objeto, denuncian e investigan las violaciones de derechos humanos y están dispuestos a poner fin a la impunidad.

Son una fuente de información fundamental sobre lo que está ocurriendo realmente en un país concreto; alertan a las organizaciones no gubernamentales internacionales y a los medios de comunicación y presentan informes a los organismos pertinentes de las Naciones Unidas y de otras organizaciones, con lo que ayudan a romper el muro de silencio que los gobiernos infractores intentan mantener:

\section{Responsabilidades de los gobiernos}

Para terminar con los abusos cometidos por los agentes encargados de la ley y el orden, Amnistía Internacional cree que todos los gobiernos deben respetar determinadas responsabilidades fundamentales.

Primero, las denuncias de violaciones de derechos humanos deben investigarse exhaustivamente. El objeto de estas investigaciones debe ser determinar las responsabilidades individuales y proporcionar una versión completa de la verdad a la víctima, a sus familiares y a la sociedad. Las investigaciones deben realizarlas instituciones imparciales, independientes de las fuerzas de seguridad, a las que se dote de la autoridad y los recursos necesarios para su labor. Los resultados de la investigación deben hacerse públicos. Cuando las violaciones de derechos humanos se han convertido en una práctica endémica en una sociedad, la investigación de los casos individuales puede no ser suficiente. Una comisión pública de investigación debe investigar el conjunto de abusos y los motivos por los que se producen. Debe examinar las instituciones y los organismos responsables y proponer cambios a la ley, las instituciones, los procedimientos y las prácticas administrativas, así como a la formación y la rendición de cuentas del personal.

Segundo, los responsables de cometer violaciones de derechos humanos deben comparecer ante la justicia. Deben rendir cuentas aunque fueran o sean agentes de un gobierno anterior $\mathrm{o}$ actual e independientemente de que sean agentes de las fuerzas de seguridad o miembros de grupos para- militares semioficiales. Las personas acusadas de crímenes contra los derechos humanos deben ser juzgadas y sus juicios deben concluir con un declaración clara de culpabilidad o inocencia. Amnistía Internacional no se pronuncia respecto a la sentencia que debe dictarse, con la condición de que no se imponga la pena de muerte. Sin embargo, la imposición sistemática de penas leves que no guardan relación con la gravedad de los delitos hace que el proceso judicial caiga en el descrédito y no sirva para impedir nuevas violaciones. El respeto de la ley no puede promoverse si los juicios no se celebran de conformidad con las normas internacionales.

Tercero, las leyes de amnistía que impiden que surja la verdad no son admisibles. Esto es válido tanto si la ley la dictan los responsables de las violaciones o los gobiernos posteriores. El interés por la reconciliación nacional después de un periodo de violencia y confusión puede lograrse mediante indultos una vez que se ha dictado sentencia. Amnistía Internacional no se pronuncia al respecto. Pero insiste en que se descubra la verdad y en que se completen los procesos judiciales.

La protección de los derechos humanos requiere actos, no palabras. Permitir que los perpetradores cometan abusos, por muy claramente que estén prohibidos por ley, sin que sufran las consecuencias, perpetúa sus delitos. Ponerlos a disposición judicial transmite a toda la sociedad el mensaje de que no va a permitirse que continúen las violaciones de derechos humanos. En la década de los años 90, la retórica de los derechos humanos ha alcanzado un relevancia sin precedentes en los asuntos mundiales. ¿En cuántos de estos países se traducirá esta retórica en medidas para terminar con la espiral viciosa de los homicidios políticos, la tortura, las «desapariciones» y otros abusos contra los derechos humanos cometidos impunemente?

\section{El caso Pinochet, una de las primeras medidas efectivas para terminar con la impunidad}

La detención, el 16 de octubre de este año, del exgeneral Pinochet en Londres, por delitos incompatibles con el respeto a la dignidad de las personas, es una de las primeras medidas que pueden ayudar a que la espiral viciosa de la impunidad deje de ser universal. En este caso, puede empezar a ser universal el concepto que se acuñó en el juicio de Nüremberg del año 1946 de que los delitos contra la humanidad los cometen personas concretas, no entidades abstractas, y son estas personas las que deben responder de sus actos ante los tribunales. No importa que hayan sido funcionarios de primer o segundo rango o ex jefes de estado.

Veinticinco años llevan esperando las víctimas del régimen de Pinochet verdad y, sobre todo, justicia. La próxima decisión de la Cámara de Los Lores será importante para éstas. Podrán testificar y esperar conocer dónde están los cientos de "desaparecidos" o, simplemente, verán que el exgeneral huye hacia la cárcel dorada de Chile donde sus tribunales y su gobierno no tienen la posibilidad ni la voluntad de juzgarlo.

¿Terminará pronto la retórica de los gobiernos de que los derechos humanos son universales excepto cuando me tocan a uno de los míos? 


\section{RESUMEN}

El hecho de que la situación política de los países se dirija hacia la consolidación democrática no implica necesariamente que cesen las violaciones a los derechos humanos, situación que sí puede ser superada cuando los índices de impunidad se reduzcan al mímino a través del fortalecimiento de un sistema internacional de justicia.

El panorama de derechos humanos en América Latina es crítico especialmente en Colombia, Brasil, Perú, México, Chile y Cuba. La implementación de estrategias como investigaciones exhaustivas e independientes, sanción a las violaciones de los derechos humanos, apoyo a los defensores de derechos humanos y respeto de los Estados a determinadas responsabilidades fundamentales es prioritario para la superación de la impunidad.

Palabras clave: Violaciones a derechos humanos, impunidad, responsabilidad estatal.

\section{ABSTRACT}

The fact that the political situation in Latin American countries is moving towards democratic consolidation, does not imply the end of human rights violations. The impunity indexes should be minimized through the strengthening of an international system of justice.

The human rights situation in Latin America is critical, especially in Colombia, Brazil, Peru, Mexico, Chile and Cuba. To address impunity, the priority is the implementation of strategies such as exhaustive and unconstrained investigations, sanctions to human rights violations, support to the defendants of human rights and state respect to specific fundamental responsibilities.

Key words: Human rights violations, impunity, state responsibility.

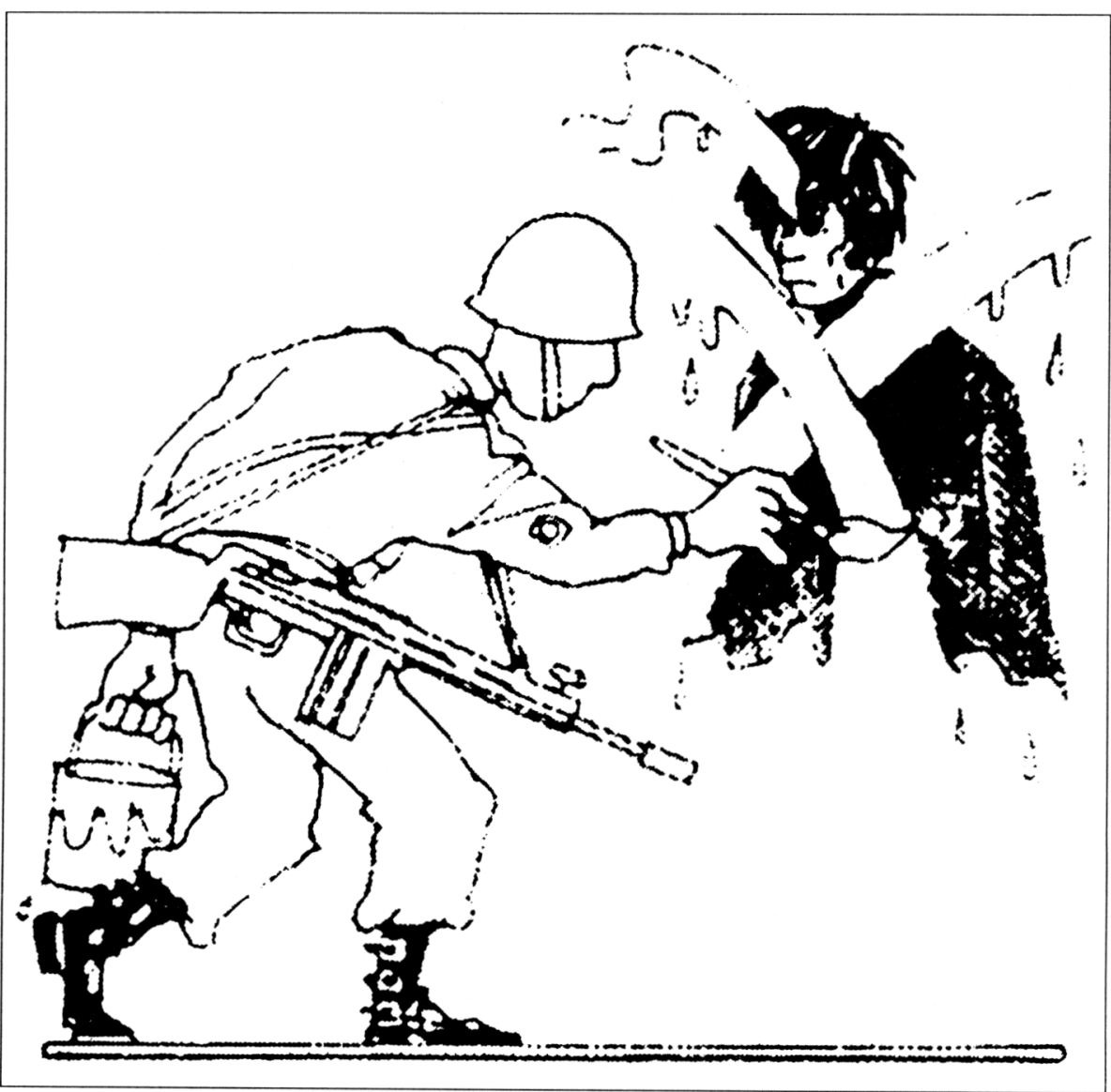

(Gentileza de "Amnistía Internacional") 